\title{
Gefangen zwischen Hilfe und Budget
}

\author{
Rainer Bernnat und Norbert Schnitzler
}

Erfahrungen der Praxis zeigen: Ökonomisierung und Erfüllung sozialer und pflegerischer Aufgaben schließen sich nicht aus. Beispiele aus der Praxis zeigen, dass dies möglich ist.

Anders als bei privatwirtschaftlichen Unternehmen steht bei sozialen Diensten die Hilfe im Vordergrund: Es geht um konkrete Unterstützung, oftmals sehr individuell ausgerichtet und zudem häufig auch mit einer emotionalen Komponente. Die Bereitstellung dieser Hilfe umfasst Tätigkeiten, die nicht nur unternehmerischen Aufgaben gleichen, sondern entsprechende Ansätze erfordern: der Betrieb eines Pflegeheims oder Krankenhauses, Fahrdienste und Versorgungslogistik für mobile Essensversorgung, Personalfragen im Kontext von Sozialstationen, um nur ein paar Beispiele zu nennen. Die ökonomische Herausforderung der sozialen Dienste liegt daher nicht schwerpunktmäßig in der Andersartigkeit ihrer Aufgaben, sondern in der zumeist dezentralen und auf althergebrachten Prinzipien, nicht modernen Managementansätzen basierenden Strukturen.

Die heutige Struktur der sozialen Dienste, insbesondere die starke Zergliederung in verschiedene Träger und deren - oftmals rechtlich unabhängigen - Unterorganisationen, machen es schwierig, einen übergreifenden Ansatz zur Optimierung der ökonomischen Leistungsposition zu verfolgen. Diese augenscheinliche Komplexität lässt sich jedoch mit vergleichsweise einfachen und zudem altbewährten Instrumenten des ökonomischen Handelns auflösen. Dazu gehören neben einer umfassenden Kostentransparenz die quantitative aber auch qualitative Zielsteuerung des Mitteleinsatzes und schließlich die Portfolio-Steuerung von Langfrist-Investitionen unter Berücksichtigung multipler Kriterien.

Kostenstransparenz führt zu erhöhtem Kostenbewusstsein und damit - geradezu automatisch - zu Kostensenkungen.

Eine pragmatische, aber wirkungsvolle Maßnahme aus dem Krankenhaussektor unterstreicht dies eindrucksvoll: Um die davon galoppierenden Kosten beim medizinischen Stationsbedarf, also von Einwegspritzen bis Wundverbänden, zu zügeln, entschlossen sich die Budgetverantwortlichen eines öffentlichen Krankenhauses dazu, die Einkaufspreise der für das Pflegepersonal frei zugänglichen Produkte an deren Entnahmeboxen anzuschreiben. Der Effekt: Der Verbrauch von sehr teuren Produkten ging schlagartig drastisch zurück. Doch flächendeckende Kos-

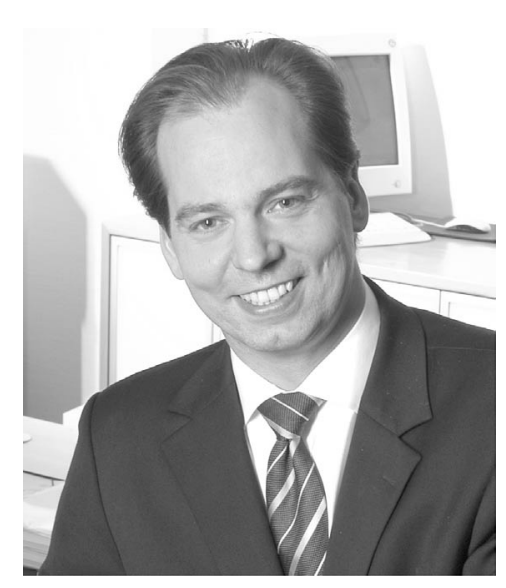

Dr. Bernnat ist Partner und Geschäftsführer der internationalen Management und Technologieberatung Booz Allen Hamilton. In seiner Funktion als Leiter des Bereichs öffentlicher Sektor im deutschsprachigen Raum betreut er Klienten und Projekte aus allen Verwaltungsebenen mit Themenstellungen zu Strategie, Organisation, Prozesse und Informationstechnologie. Vor seiner Zeit bei Booz Allen Hamilton hat er bei der IBM in Deutschland und im europäischen Ausland in den Bereichen Beratung, Vertrieb, Marketing und Management gearbeitet. Er ist Mitglied des Vorstands der Initiative D21, Mitglied des Präsidiums der Special Olympics und in mehreren Beiräten tätig. Er hat an der Universität Frankfurt am Main sowie an der Universität Pamplona in Spanien studiert und promoviert.

tentransparenz stellt weit mehr dar, als ein Instrument zur Bedarfszügelung. Für Ansätze zur strukturellen Kostensenkung ist sie ein erster Schritt, um die Kostentreiber zu identifizieren, deren Beeinflussung nachhaltige Verbesserungen erwarten lassen.

Ein weiteres Beispiel aus der Praxis eines freigemeinnützigen Trägers von Krankenpflegediensten und anderen Sozialeinrichtungen. Unter dem Druck der drohenden Insolvenz entschloss sich dieser zur Durchführung einer von externen Beratern unterstützten Wirtschaftlichkeitsanalyse. Sehr schnell wurde klar: Die Führungsgremien 


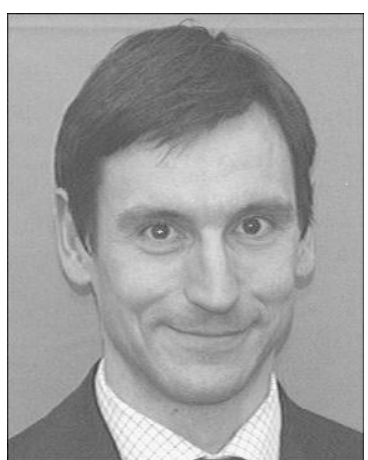

Norbert Schnitzler ist Senior-Projektleiter bei Booz Allen Hamilton. Seine Beratungsschwerpunkte liegen im Sozial- und Gesundheitswesen. Im Mittelpunkt stehen Fragen der allgemeinen Verwaltungsmodernisierung und insbesondere der Zukunftssicherung öffentlicher Leistungserbringung im Sozialbereich. Zu seinen Kunden zählen deutsche und europäische Ministerien sowie Krankenhäuser und Pflegeeinrichtungen verschiedener Ausrichtung. Nach seinem Magisterabschluss in Politologie an der Universität Bonn absolvierte er im Rahmen des McCloy-Stipendiums der Studienstiftung des deutschen Volkes ein zweijähriges Master-Programm in Public Administration an der Harvard University in den USA.

hatten keine ausreichende Transparenz darüber, wie sich Kosten und Erlöse auf die einzelnen Aktivitäten verteilten. Anders ausgedrückt: Es war offensichtlich, dass es ein Missverhältnis zwischen Kosten und Erlösen gab; es war aber nicht ersichtlich, wo im weit verzweigten Netz der Kostenpositionen und Erlösquellen diese Diskrepanz vorlag und in welchen Bereichen möglicherweise wirtschaftlich, also kostendeckend gearbeitet wurde.

Der Zustand dieser Organisation glich einem Schiff, das aufgrund eines Lecks zu sinken drohte, keiner wusste jedoch, wo sich das Leck befand, das es zu stopfen galt. Auf Basis einer strukturierten Kosten- und Erlösanalyse war bereits nach wenigen Wochen klar, in welchen Bereichen die Verluste erwirtschaftet wurden. Die Formulierung von geeigneten Maßnahmen zur Abwendung des wirtschaftlichen Kollapses war dann eine vergleichsweise leichte Übung.

Transparenz ist die Basis für gezieltes Handeln. So ist Kostentransparenz eine notwendige Voraussetzung für effektives Kostenmanagement. Ein wichtiger Tätigkeitsbereich der Wohlfahrtsverbände sind Krankenhäuser und andere Pflegeeinrichtungen. Für diese gilt, was aktuell für alle Krankenpflegeeinrichtungen gilt: Nur durch ein stringentes Kostenmanagement in Kombination mit einer zukünftig bewussten Wahl des eigenen Leistungsportfolios wird es möglich sein, unter den gegebenen wirtschaft- lichen Prämissen überlebensfähig zu sein. Für die meisten Häuser gilt: Durch ein stringentes und strategisch ausgerichtetes Einkaufsmanagement können bereits 30 bis 50 Prozent der aktuell ermittelten Unterdeckung beseitigt werden.

Kostentransparenz bildet auch eine essenzielle Voraussetzung beim Redesign der Prozesse und der sich anschließenden Restrukturierung der Ausstattung mit Informationstechnologie (IT). Wie in den meisten Institutionen des öffentlichen Sektors sind sowohl Abläufe als auch Informationstechnologie der sozialen Dienste über einen langen Zeitraum gewachsen. Die Arbeitsverrichtung in den Leistungsbereichen und in der Sachbearbeitung ist oftmals gekennzeichnet durch einen hohen Anteil sequenziell ablaufender, papierbasierter Prozesse mit vielfältigen, teilweise komplexen Schnittstellen innerhalb der Organisationen und zu externen Partnern. Daneben finden sich häufig starre Kapazitätssteuerungssysteme, welche eine flexible Arbeitsverteilung über diverse Sachbearbeitungs- oder Leistungsgruppen hinweg nur sehr eingeschränkt zulassen.

Die informationstechnologische Unterstützung der Geschäftsprozesse weist vielfältige Einschränkungen auf, wie die fehlende fallabschließende Abbildung von Prozessen, eingeschränkte Arbeitsvorrats- und Workflow-Steuerung, Probleme beim parallelen Zugriff auf Vorgänge und bei der externen Datenübertragung. Somit wird eine effiziente und zügige Vorgangsbearbeitung aus einer Hand erheblich eingeschränkt.

Die signifikanten Effizienzreserven in der Arbeitsverrichtung können in zwei Schritten erschlossen werden: Erstens müssen in einer Bestandsaufnahme die Ist-Prozesse einschließlich des dazugehörenden Mengengerüsts erhoben und in ihrer Bedeutung eingeordnet werden. Im Anschluss werden zweitens die Soll-Prozesse unter Berücksichtigung der IT-Anforderungen modelliert. Die Quantifizierung der Kosten- und Nutzenpotenziale eines Soll-IstVergleichs bei verschiedenen Migrationsszenarien gibt Aufschluss über den ökonomischen Nutzen des Vorhabens.

In der Regel wird ein Großteil der Kapazität durch wenige Prozesse gebunden - diese Prozesse sind gezielt und zu einem frühen Zeitpunkt zu optimieren, um einen wahrnehmbaren Nutzen schon in frühen Phasen zu erreichen. Erfahrungswerte aus Projekten des öffentlichen Sektors zeigen: Gerade in der Sachbearbeitung können 30 bis 40 Prozent der Kosten durch gezielte Optimierung eingespart werden - Einsparungen, die den sozialen Diensten direkt zufließen könnten.

Eine Ökonomisierung trägt zur langfristigen Sicherung und zum weiteren Ausbau der sozialen Dienste als eine der tragenden Säulen unserer Gesellschaft bei. Wirtschaftliches Handeln steht dem sozialen Auftrag nicht entgegen - es bildet vielmehr eine wichtige Voraussetzung für einen zielgerichteten Einsatz von Ressourcen in Bereiche, die direkt den Leistungsempfängern zugute kommen. 


\section{Praxisbeispiel: Bürokratie in der Pflege}

Eine elektronische Tätigkeitsanalyse kann schnell den Überblick über die tatsächlichen Arbeitsabläufe verschaffen. Mit diesem Verfahren lassen sich auch Spitzen- und Überlastungszeiten entzerren.

Ein Arbeitstag in der Pflege, das sind unzählige Details und Handgriffe. Sie reichen von Medikamente und Mahlzeiten verteilen, Bewohner in den Essenssaal fahren, mit ihnen und deren Angehörigen reden, bis zum schriftlichen Festhalten der therapeutischen Maßnahmen sowie dem Planen und Besprechen der Dienste.

Dies ist nur ein kleiner Ausschnitt von einem Tagesablauf, bei dem die Bürokratie langsam überhand zu nehmen droht. Denn immer mehr Mitarbeiter in der Pflege klagen über zu viel Verwaltung und Dokumentation. Häufig stellen sie sich die Frage »Wie viel unserer Zeit entfällt eigentlich noch auf die Pflege und pflegetherapeutische Arbeiten? « Leitungen und Controller in großen Einrichtungen, wie Seniorenheimen, wollen immer genauer wissen: »Welche Tätigkeiten erledigt ein Mitarbeiter im Laufe eines Arbeitstages? In welchem Umfang werden die Aufgaben von Fachkräften und von Pflegehilfskräften ausgeführt?«

Um Klarheit in diesen Fragen zu bekommen, ließ die AWOcura gGmbH in Duisburg für ihre Seniorenzentren eine elektronische Tätigkeitsanalyse durchführen, ein Verfahren, wie es beispielsweise contec Gesellschaft für Organisationsentwicklung mbH seit fünf Jahren anbietet.

Für die Analyse in den Seniorenzentren der AWOcura wurden einen Monat lang, für jeweils einen Pflegewohnbereich die Zeiten und Tätigkeiten erfasst. Jeder Mitarbeiter gab mithilfe eines Mini-Computers ein, wann, wo und was von ihm erledigt wurde. Der Mini-Computer entspricht der Größe eines PocketPCs. Durch diese Erfassung werden die klassische Selbstaufschreibung durch die Mitarbeiter und die elektronische Datenverarbeitung in einem System verbunden. Um die Handhabung der Erfassungsgeräte zu vereinfachen, konnten die Tätigkeiten anhand einer vorgegebenen codierten Tätigkeitsliste ausgewählt werden. Diese Liste wurde in einem Workshop von Mitarbeitern sowie Team- und Pflegeleitungen der Seniorenzentren der AWOcura gGmbH erarbeitet. Der Mitarbeiter kann die Tätigkeiten über die Tastatur des Mini-Computers oder über einen integrierten Barcodeleser auswählen und erfassen. Die Erfassungszeit der Mitarbeiter zur Tätigkeitsdokumentation betrug drei Prozent der Arbeitszeit.

In einem zweiten Schritt wurden die Daten von einem wissenschaftlichen Forschungsinstitut anonymisiert ausgewertet. Auf diese Weise konnten detaillierte Erkenntnisse über die internen Prozessabläufe, Spitzenbelastungen und Optimierungsmöglichkeiten gewonnen werden.

Die Ergebnisse lagen Ende 2005 vor und wurden von der contec GmbH präsentiert. Unabhängig von den
Ergebnissen im Einzelfall war für die Geschäftsführerin der AWOcura gGmbH, Wilma Katzinski, wichtig, dass die eigene Arbeit objektiv eingeschätzt werden konnte. Anhand eines Plausibilitätsvergleichs kam die contec GmbH zu dem Ergebnis, dass in den untersuchten Seniorenzentren insgesamt effizient gearbeitet wurde. Auch die Quote an Pflegefach- und Pflegehilfskräften entsprach der im Pflegebereich Üblichen. Interessant war jedoch, so Sascha Sassen von der contec $\mathrm{GmbH}$, dass innerhalb der Wohnbereichsgruppen der Seniorenzentren die Arbeit sehr unterschiedlich organisiert ist. Aufgaben und Tätigkeiten wurden von den Fach- und Hilfskräften in teilweise deutlich verschiedenen Umfängen erledigt. Das Für und Wider der Differenzen in der Aufgabenteilungen wird nun mit den Mitarbeitern geklärt. Auf diese Weise erhofft sich die Geschäftsführung der AWOcura Ansätze, die Arbeit weiter zu optimieren, Spitzen- und Überlastzeiten zu entzerren und Fachkräfte von fachfremden Tätigkeiten zu entlasten.

Auch in den Seniorenzentren der AWOcura, hatten die Mitarbeiter das Gefühl, dass im Arbeitsalltag die Pflege ab- und die Büroarbeiten zunehmen. Entsprechend unzufrieden sind viele mit ihrer Arbeitssituation. Zu dieser Fragestellung ergab die Analyse, dass der Anteil der Zeiten für Verwaltung lediglich bei fünf und für Dokumentation zehn Prozent liegt.

Viele Mitarbeiter empfinden die Bürokratie in ihrem Arbeitsalltag als zu hoch, erklärt Sascha Sassen von der contec Gmbh, weil Tätigkeiten, die dem Bewohner nicht direkt zugute kommen, oft mit Verwaltungsaufgaben gleichgesetzt werden. Somit wurde auch das Bestellen und Verteilen von Medikamenten von den Pflegekräften als Verwalten eingestuft, eine Tätigkeit, die zwar nur indirekt mit dem Bewohner zu tun hat, aber trotzdem dazu gehört.

Der Durchführung der elektronischen Tätigkeitsanalyse musste die Mitarbeitervertretung zustimmen. Dies erforderte einige Überzeugungsarbeit, da Rationalisierungen und Kündigungen seitens der Mitarbeiter befürchtet wurden. Die Einbindung der Mitarbeiter durch einen Workshop und ihre Einarbeitung durch die contec $\mathrm{GmbH}$ führte zu einer breiten Akzeptanz.

Ute Arentzen

contec GmbH, Sinterstraße 8a, 44795 Bochum, Telefon 0234 452730, Fax 0234 4527399, E-Mail

info@contec.de,

Internet http://www.contec.de

AWOcura Duisburg, Pulverweg 23, 47051 Duisburg, Telefon 0203 3095548, E-Mail Katzinski@awocura.de, Internet http://www.awocura.de 